\title{
Schwangerschaft transgeschlechtlicher Personen: eine Herausforderung für die Frauenheilkunde und Geburtshilfe
}

Vor 2011 musste sich eine Person nach dem Transsexuellengesetz (TSG) sterilisieren lassen, wenn sie in einem anderen als ihrem Geburtsgeschlecht leben und entsprechend ihren Vornamen und Personenstand ändern wollte. Das TSG existiert noch, der Zwang zur Sterilisierung wurde jedoch vom Bundesverfassungsgericht für verfassungswidrig erklärt; der entsprechende Paragraf wird seitdem nicht mehr angewendet. Der kleinen, aber wachsenden Gruppe von Menschen in unserer Gesellschaft, die sich als „trans“ versteht, eröffnen sich dadurch neue Möglichkeiten. Mit dem Begriff „trans“ bzw. „Transidentität“ wird mittlerweile eine Vielfalt von Lebensentwürfen und -identitäten erfasst. Auch Kinderwunsch und Schwangerschaft können Teil dieser Lebensentwürfe sein.

Seit 2011 ist es auch Transmännern möglich, ihren Kinderwunsch durch eine eigene Schwangerschaft zu verwirklichen. Die Frauenheilkunde und Geburtshilfe stehen damit vor einer ungewöhnlichen Herausforderung. Ein klinisches Gebiet, welches sich traditionell der Frauengesundheit widmet, muss sich für andere geschlechtliche Identitäten öffnen, wenn eine flächendeckende und qualitativ hochwertige Gesundheitsversorgung gewährleistet werden soll. Dies macht es erforderlich, sich zunächst einmal mit den medizinischen, sozialen und ethischen Auswirkungen des Phänomens vertraut zu machen.

Der Begriff „trans“ bedeutet, vereinfacht ausgedrückt, dass sich Personen mit dem bei ihrer Geburt eingetragenen Geschlecht nicht identifizieren. Transpersonen können sich dem jeweils anderen Geschlecht zugehörig fühlen, d.h. eine Person, die mit Uterus und Eierstöcken geboren wurde, kann sich als Mann fühlen und durch Hormontherapie und geschlechtsmodifizierende Operationen dem bevorzugten Geschlecht annähern. Gerade unter jüngeren Transpersonen finden sich aber auch viele, die sich als abinär bezeichnen und die gesellschaftliche und biologische Dichotomie in Mann und Frau überwinden wollen. Verwirrend kann die Vielzahl an Begriffen wirken, die dem Phänomen gerecht werden wollen. Transsexualität, Transidentität und Transgeschlechtlichkeit wurden und werden für und von Transpersonen verwendet. Allerdings wird von der Verwendung des Begriffs Transsexualität oft abgeraten, da er zu der falschen Annahme führen kann, dass Trans-Sein etwas über die Sexualität der Person aussagt.

Vereinzelt wurde mittlerweile über Schwangerschaften von Transmännern berichtet. Dem Fachgebiet der Frauenheilkunde und Geburtshilfe, das schon dem Namen nach eigentlich Frauen behandelt, stellt sich die Frage, wie es angemessen auf die Bedürfnisse dieser Personen eingehen kann. Es sind ganz praktische Fragen, die beantwortet werden müssen: Welche medizinischen Besonderheiten stellen sich im Schwangerschaftsverlauf und bei der Entbindung? In welchen Zimmern können Transmänner auf der Wochenbettstation unterkommen? Wie werden die betroffenen Personen angesprochen? Auch das Recht geht bisher nicht auf solche neuen Familienkonstellationen ein. Männer, die gebären, werden immer noch mit ihrem alten, weiblichen Namen in die Geburtsurkunde eingetragen - dem Namen einer juristischen Person also, die eigentlich nicht mehr existiert.

Solche Fragen werden mittlerweile auf internationaler Ebene vermehrt diskutiert. Die internationale Konferenz „Trans Pregnancy“, die vom 14. bis 16. Januar 2020 in Leeds (England) stattfand, war der Abschluss eines 3-jährigen internationalen Forschungsprojekts, das sich mit rechtlichen, ethischen und sozialen Aspekten von Transschwangerschaften auseinandersetzte. Eingeladen war auch Freddie
McConnell, ein britischer Journalist, dessen Schwangerschaft große mediale Aufmerksamkeit erlangt hatte und der im letzten Jahr vergeblich für die rechtliche Anerkennung als Vater geklagt hatte. McConnell wurde bekannt, da er seine Schwangerschaft im Rahmen der filmischen Dokumentation „Seahorse“ begleiten ließ. Auf der Konferenz wurden u. a. Beispiele vorgestellt, wie eine transinklusive Medizin gestaltet sein könnte. In Brighton (England) haben sich seit einiger Zeit die „Gender Inclusion Midwives“ auf die Betreuung von Schwangerschaften von Transpersonen spezialisiert. Neben der medizinischen Betreuung bieten sie auch Bildungsprogramme an und entwickeln Leitfäden, die helfen sollen, den Klinikalltag diskriminierungsfrei zu gestalten.

Im deutschsprachigen Raum gibt es bisher keine Forschung zum Thema Transschwangerschaft. Ein Pilotprojekt am Institut für Ethik und Geschichte der Medizin der Universitätsmedizin Göttingen soll nun die praktischen Schwierigkeiten erkunden, mit denen Gesundheitsversorger und betroffene Personen konfrontiert sind. Ziel ist eine erste Erfassung der Herausforderungen für die Frauenheilkunde und Geburtshilfe bzw. für das Gesundheitswesen im Allgemeinen, um daraus Empfehlungen für eine Anpassung der medizinischen Versorgung ableiten zu können.

Ärztinnen und Ärzte sowie Hebammen, die schon Erfahrungen mit dem Kinderwunsch oder einer Schwangerschaft von Transpersonen gemacht haben, sind herzlich eingeladen, sich an unserer Befragung zu beteiligen. Bei Interesse wenden Sie sich dazu bitte an Frau Prof. Claudia Wiesemann (cwiesem@gwdg.de).

\section{Interessenkonflikt}

Die Autorinnen/Autoren geben an, dass kein Interessenkonflikt besteht. 


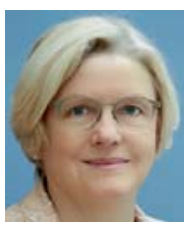

\section{Claudia Wiesemann}

Prof. Dr. med., Direktorin des Instituts für Ethik und

Geschichte der Medizin

an der Universitätsmedizin

Göttingen, Mitglied

des Deutschen Ethikrats

2012-2020

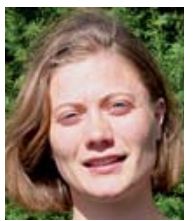

\section{Hanna Marla Frentz}

cand. med., Institut für

Ethik und Geschichte

der Medizin, Universitäts-

medizin Göttingen

Korrespondenzadresse

Prof. Dr. Claudia Wiesemann

Institut für Ethik und Geschichte der Medizin

Universitätsmedizin Göttingen

Humboldtallee 36

37073 Göttingen

cwiesem@gwdg.de

Bibliografie

DOI https://doi.org/10.1055/a-1125-8372

Geburtsh Frauenheilk 2020; 80: 670-671 @ Georg

Thieme Verlag KG Stuttgart · New York I

ISSN 0016-5751 